\title{
Cambios estructurales en índices bursátiles del mercado MILA entre los años 2008 y $2018^{*}$
}

\author{
Recibido: 12 de febrero de 2020 - Aprobado: 17 de abril de 2020 \\ https://doi.org/10.22395/seec.v23n54a2
}

\author{
Nicolás Morales León ${ }^{* *}$ \\ José Rodrigo Vélez Molano**
}

\section{RESUMEN}

El presente trabajo identifica las posibles fechas de cambios estructurales en los mercados bursátiles que conforman el MILA y sus posibles precursores. Mediante el uso de pruebas clásicas de estabilidad y un estadístico robusto para modelos con heterocedasticidad, se realiza la comparación entre ambas metodologías en las series diarias de los retornos, del precio y el cambio porcentual del volumen de índices accionarios. Para los modelos de los retornos, las fechas de cambio estructural coinciden con eventos de alcance internacional ocurridos en Estados Unidos y Europa, mientras que para los modelos de la variación porcentual del volumen las fechas están en línea con el desempeño de la economía local.

\section{PALABRAS CLAVE}

Cambio estructural; mercados financieros y macroeconomía; bootstrap.

\section{CLASIFICACIÓN JEL}

$\mathrm{C} 22, \mathrm{E} 44, \mathrm{C} 15$.

\section{CONTENIDO}

Introducción; 1. Marco de referencia; 2. Metodología; 3. Resultados; 4. Conclusiones; Bibliografía.

\footnotetext{
* El presente artículo es el resultado de la investigación desarrollada en el marco del proyecto Modelación de los mercados financieros de Colombia adscrito al Grupo de Investigación en Estudios Sociales, Financieros e Internacionales -ESFI — (clasificado en categoría C en Colciencias en el año 2018), financiado por la Universidad de la Salle y realizado entre abril de 2018 y noviembre de 2019. Artículo basado en el trabajo de grado obligatorio para optar al título de Profesional en Finanzas y Comercio Internacional de la Universidad de La Salle.

•. Profesional en Finanzas y Comercio Internacional, Universidad de La Salle, Bogotá, Colombia. Correo electrónico: nmorales61@unisalle.edu.co

-. Economista y magíster en Economía, Universidad Pontificia Javeriana, Bogotá, Colombia. Profesor de Planta Programa de Finanzas y Comercio Internacional, Universidad de La Salle, Bogotá, Colombia. Correo electrónico: jrvelez@unisalle.edu.co
} 


\title{
STRUCTURAL CHANGES IN THE MILA STOCKMARKETS INDICATORS BETWEEN 2008 AND 2018
}

\begin{abstract}
This article identifies the possible dates for structural changes in stock markets conforming the MILA and its possible pioneers. Through classic stability testing and a robust statistic for models with heteroscedasticity, this research performs a comparison between both methodologies in daily timeseries of returns and a percentage change in the volume of stock indicators. For the return models, the structural change dates coincide with international scope events in Europe and the United States, whilst for the percentage variation of volume models, dates are aligned with the performance of the local economy.
\end{abstract}

\section{KEY WORDS}

Structural change; stock markets and macroeconomics; bootstrap.

\section{JEL CLASSIFICATION}

C22, E44, C15

\section{CONTENT}

Introduction; 1. Reference framework; 2. Methodology; 3. Results; 4. Conclusions; bibliography

\section{MUDANÇAS ESTRUTURAIS EM ÍNDICES DO MERCADO DE AÇÕES MILA ENTRE 2008 E 2018}

\section{RESUMO}

Este trabalho identifica as possíveis datas de mudanças estruturais nos mercados de ações que conformam o MILA e seus possíveis precursores. Por meio do uso de testes clássicos de estabilidade e de estatística robusta para modelos com heterocedasticidade, é realizada a comparação entre ambas as metodologias nas séries diárias dos retornos do preço e da mudança porcentual do volume de índices acionistas. Para os modelos dos retornos, as datas de mudança estrutural coincidem com eventos de abrangência internacional ocorridos nos Estados Unidos e na Europa, enquanto para os modelos da variação porcentual do volume, as datas estão de acordo com o desempenho da economia local.

\section{PALAVRAS-CHAVE}

Mudança estrutural; mercados financeiros e macroeconomia; bootstrap.

\section{CLASSIFICAÇÃO JEL}

$\mathrm{C} 22, \mathrm{E} 44, \mathrm{C} 15$

\section{CONTEÚDO}

Introdução; 1. Referencial teórico; 2. Metodologia; 3. Resultados; 4. Conclusões; Bibliografia. 


\section{INTRODUCCIÓN}

El presente trabajo de investigación se centra en la identificación de cambios estructurales en los principales índices bursátiles de los países que conforman el Mercado Integrado Latinoamericano (MILA) entre los años 2008 y 2018. Dadas las características econométricas de este fenómeno, se plantea una alternativa de especificación del quiebre para los modelos de series de tiempo que explican el comportamiento de los índices bursátiles.

La detección de cambios estructurales es de suma importancia para determinar qué eventos pueden provocar una alteración en la especificación de los modelos de predicción. De este modo, se puede definir si es necesario estimar nuevos modelos que tengan en cuenta el quiebre, ya sea modificando variables y estimadores o agregando variables que permitan diferenciar los periodos, como lo hacen las variables dicotómicas. Además, conocer la existencia del cambio estructural permite modificar los modelos en las fechas adecuadas para mejorar la capacidad de pronóstico y disminuir el riesgo de estimación de resultados erróneos.

Según Pérez (1995), los cambios estructurales desde el punto de vista econométrico son definidos como una alteración de los modelos que habitualmente son utilizados para representar un sistema económico, es decir, un cambio estructural se presenta cuando existe alguna modificación en los parámetros a lo largo de una serie temporal de observaciones. Como lo sugieren Turtle y Zhang (2014), en series de tiempo económicas y financieras, es habitual encontrar cambios de especificación debido a la dinámica existente. De este modo, las series de tiempo generalmente presentan inestabilidad y, por tanto, cambios estructurales.

Narayan, Narayan y Mishra (2013) relatan la existencia de investigaciones que examinan el comportamiento de los precios en los mercados bursátiles, sin embargo, las investigaciones de cambios estructurales en estos son escasas. En contraste, sí hay evidencia de otras investigaciones aplicadas al análisis de variables macroeconómicas, como las tasas de desempleo o de crecimiento del PIB cuyos parámetros suelen cambiar en el tiempo (Hansen, 2001). Por su parte, Shahani y Subhan (2018) proponen analizar el cambio estructural lo más cerca de un evento de impacto en los mercados bursátiles.

Con la entrada en vigor del MILA, los mercados de renta variable de los países pertenecientes pueden estar sujetos a choques comunes y específicos que no eran visibles o medibles antes de la puesta en marcha del esquema. Según Pérez (1995), dichos choques se pueden entender como una ruptura en el modelo que explica 
el comportamiento de una serie económica, siendo plausible la existencia de un cambio estructural en la fecha en la cual el MILA empezó a operar.

En consecuencia, el problema consiste en identificar la existencia de un cambio de especificación de los modelos que explican los índices bursátiles de los países que conforman el MILA en las fechas en las cuales se presentó algún suceso importante, ya fuera político, económico o social, de índole nacional o internacional, que provocara modificaciones en la estructura de los mercados.

Para tal fin, se construyen modelos econométricos capaces de explicar el comportamiento de los retornos del precio y la variación porcentual del volumen de cada índice, para luego someterlos a las correspondientes pruebas de cambio estructural. Las pruebas de detección del cambio seleccionadas son aquellas pruebas clásicas disponibles en el software E-Views 10, así como una metodología con un proceso más robusto dadas las condiciones de heterocedasticidad de los modelos. Finalmente, una vez localizados los puntos de cambio estructural, se hace un breve análisis de los eventos que tuvieron lugar en las fechas de quiebre de cada modelo.

La muestra seleccionada comprende el periodo entre 2008 y 2018. Las variables utilizadas son los retornos logarítmicos diarios de los principales índices bursátiles de los países que conforman el MILA, estos son, el índice Colcap para Colombia, el índice de precios selectivo de acciones (IPSA) para Chile, el índice de precios y cotizaciones (IPC) para México y el SEP/BVL para Perú. Asimismo, se utiliza el cambio porcentual del volumen diario transado. Por su parte, la variable independiente para los modelos de series de tiempo fue el índice Dow Jones Industrial, otras variables se tuvieron en cuenta, pero no resultaron ser significativas.

Este artículo se organiza de la siguiente manera. En la primera sección se presenta el marco de referencia en el cual se realiza una breve revisión de literatura y se explica qué es un cambio estructural y algunas de las pruebas que se utilizan para su detección. En la segunda sección se explica la metodología y las variables utilizadas para la investigación. La sección tres presenta los resultados. Finalmente, se desarrollan las conclusiones.

\section{MARCO DE REFERENCIA}

\subsection{Revisión de literatura}

De acuerdo con Anderson y Mizon (1989), el concepto de cambio estructural, desde el punto de vista estadístico, se relaciona con la estructura del modelo que trata de explicar el comportamiento de las series económicas, más específicamente el 
cambio en las relaciones existentes entre las variables explicativas y la variable dependiente dentro de un modelo de series de tiempo.

Por su parte, para MacKinnon (1988), dicho cambio presupone que el modelo puede ser dividido en dos o más submuestras, y para cada una de ellas los estimadores del modelo de regresión serán diferentes. La división puede corresponder a distintos regímenes económicos o políticos, como una modificación en el tipo de cambio de fijo a flotante o una fuerte política contractiva.

Como Anderson y Mizon (1989) señalan, los cambios estructurales son de gran utilidad para la economía, pues ayudan a evaluar qué tan apropiados y estables son los modelos. Además, Shahani y Subhan (2018) concluyen que la detección de un cambio estructural evita estimaciones incorrectas debido al cambio en los parámetros o permite descubrir la influencia de variables exógenas que afectan el comportamiento de los modelos, por ejemplo, un cambio dada la gran depresión, la crisis del 2008 o la crisis de Asia Pacífico.

Según Hillebrand (2005), en series de tiempo las inferencias erróneas son comunes para los modelos ARMA ya que estos pueden llegar a ser estimados con el componente GARCH sin que sea necesario. Esta estimación se hace habitualmente cuando no se identifica un cambio estructural en el debido momento dentro de la serie. Al considerar que los parámetros, la media, la varianza y las tendencias suelen cambiar en modelos de series macroeconómicas y financieras, se tiende a estimar modelos de volatilidad condicional sin considerar, en primera instancia, que pueden ser consecuencia del cambio de estructural.

Ahora bien, identificar el tipo de cambio estructural es crucial para la especificación de un modelo que responda a las condiciones cambiantes de un análisis económico y financiero. Para esto, Anderson y Mizon (1989) proponen las siguientes categorías: i) las alteraciones puntuales, que son aquellos cambios en los cuales los valores de los parámetros se modifican, pero retornan a sus valores anteriores una vez superado el evento; ii) de épocas específicas en donde el cambio es determinista y no representa en sí una dificultad en su estimación, pues son conocidos los factores que permiten su existencia; iii) regímenes diferenciados, este tipo de cambio estructural puede ser estocástico y en él se alteran los valores de los parámetros así como el conjunto de variables explicativa; finalmente, iv) el proceso en el cual los parámetros van sufriendo un cambio evolutivo.

Una revisión de literatura interesante la realiza Pérez (1995) con un recorrido por las diferentes pruebas que existen para determinar la presencia de un cambio estructural. En este sentido, los divide de la siguiente forma: los basados en la suma cuadrática de 
los errores, como los propuestos por Chow (1960) y Quandt (1958); los contrastes de restricción paramétrica aplicados al cambio estructural, como el test Wald o el ratio de verosimilitud; los contrastes no paramétricos, como los test basados en estadísticos de tipo Wilcoxon y medianas; finalmente, las pruebas basadas en estimaciones recursivas como los test Cusum.

Sin embargo, algunas de estas pruebas son paramétricas y no permiten modelos con problemas de autocorrelación o heterocedasticidad, características comunes en los modelos de series financieras. Por esto, según Pérez (1995), surgen varios tipos de modelos no paramétricos que evitan asumir una distribución; lo que pretenden es determinar si alguna de las variables de la muestra pertenece o no a la distribución de los datos sin tomar una distribución a priori.

Estudios recientes, como el de Shahani y Subhan (2018), proponen analizar el cambio estructural lo más cerca de un evento de impacto, en el cual se esperaría que este exista para los principales índices bursátiles de cinco países con mercados de capitales que manejan un volumen diario considerable en la Unión Europea (Reino Unido, Francia, Alemania, Finlandia y España). Los autores usan como metodología las pruebas de Cusum y Cusumo con el fin de identificar las posibles fechas de existencia del cambio estructural, pero sin descartar la clásica prueba de Chow para confirmar que efectivamente existen.

\subsection{Pruebas de cambio estructural}

Como Pérez (1995) expone, una de las primeras pruebas, y posiblemente la más conocida para la detección del cambio estructural, es la prueba propuesta por Gregory Chow en 1960, conocida como la prueba de Chow. Esta prueba consiste en dividir la muestra en dos submuestras. Luego se compara la suma de errores al cuadrado de la muestra completa con la suma de errores al cuadrado obtenidas de cada una de las submuestras, como se aprecia en la ecuación [1].

$$
F=\frac{\frac{R S S R-\left(U S S R_{1}+U S S R_{2}\right)}{k}}{\frac{U S S R_{1}+U S S R_{2}}{n-2 k}}
$$

Donde RSSR es la suma de errores al cuadrado de la regresión completa, USSR $_{i}$ es la suma de errores al cuadrado de cada una de las submuestras, $k$ es el número de parámetros en la ecuación y $n$ es el tamaño de la muestra. Así, Chow (1960) muestra que se obtiene un $F$ estadístico con una distribución $F$ con $(k, n-2 k)$ grados de 
libertad para contrastar la hipótesis nula de la no existencia de cambio estructural en la fecha dada.

No obstante, para aplicar esta prueba se debe conocer la fecha o momento en el que se presentó el cambio estructural de la serie, lo cual no siempre es el caso. Para ello, Andrews (1993) plantea el desarrollo de iteraciones para la prueba de Chow, en el cual el cambio estructural existe en el valor máximo de $F$. Se divide la muestra cuantas veces sea necesario y se aumenta una observación a la vez. Las iteraciones deben ocurrir entre el $15 \%$ y el $85 \%$ de los datos para que existan valores suficientes dentro de cada submuestra, lo cual arroja resultados más potentes, el planteamiento se muestra en la ecuación [2].

$$
\begin{gathered}
\text { Max.F }=(F(\tau)) \\
\tau_{1} \leq \tau \leq \tau_{2}
\end{gathered}
$$

Donde $F$ es el estadístico de Chow y $\tau$ es cada uno de los puntos entre los límites del $15 \%$ y $85 \%$ de la muestra denotados por $\tau_{1}$ y $\tau_{2}$ respectivamente. Asimismo, el estadístico se contrasta en una distribución Chi cuadrado que establece un valor crítico más alto o, como lo plantea Quandt (1958), el valor $F$ puede ser calculado como el Likelihood Ratio y se ajusta a esta distribución.

Posteriormente, Bai y Perron (1998) desarrollaron diferentes métodos para determinar si existe más de un cambio estructural en la serie de análisis. El primero es la optimización global, la cual se enfoca en obtener la mínima suma de errores al cuadrado cambiando la cantidad de quiebres $(m)$ dentro del modelo, como aparece en la ecuación [3].

$$
S(\beta, \delta \mid\{T\})=\sum_{j=0}^{m}\left(\sum_{t=T_{j}}^{T_{j+1}-1} y_{t}-X_{t}^{\prime} \beta-Z_{t}^{\prime} \delta_{j}\right)^{2}
$$

Donde $m$ representa la cantidad de potenciales quiebres, $\{\mathrm{T}\}_{\mathrm{m}}=\left(\mathrm{T}_{1}, \ldots, \mathrm{T}_{\mathrm{m}}\right)$ como el conjunto de las fechas donde ocurre cada quiebre y $T_{j}$ hasta $T_{j+1-1}$ en el régimen $j=0, \ldots, m$ representan las observaciones entre los puntos de quiebre. Además, los autores dividen las variables en dos grupos: las variables $X$ son aquellas que se presume sus parámetros se mantienen constantes, y las variables $Z$ cuyos parámetros varían entre regímenes. Los parámetros de las variables $X$ y $Z$ están representados por $\beta$ y $\delta$ respectivamente.

El siguiente método consiste en una generalización de la prueba de QuandtAndrews para determinar la igualdad de $\delta_{j}$ en múltiples regímenes. Según Bai y Perron (2003), se desarrolla bajo un $F$ estadístico con hipótesis nula de la no existencia de 
un cambio estructural contra una alterna de la existencia de $l$ quiebres en la serie, es decir, $H_{\circ}, \delta_{0}=\delta_{1}, \ldots, \delta_{1+1}$.

$$
F(\delta)=\frac{1}{T}\left(\frac{T-(l+1) q-p}{k q}\right)(R \delta)^{\prime}\left(R \mathcal{V}(\delta) R^{\prime}\right)^{-1} R \delta
$$

Donde $\delta$ representa al estimador que minimiza los errores al cuadrado con $l$ quiebres, $\mathrm{R}$ es una matriz tal que $(\mathrm{R} \delta)^{\prime}=\left(\delta 0^{\prime}-\delta 1^{\prime}, \ldots, \delta l^{\prime}-\delta 1+1^{\prime}\right)$ y $\mathrm{V}(\delta)$ es la matriz de varianza covarianza de $\delta, p$ es el largo del vector $X$ y $q$ el largo del vector $Z$. De acuerdo con Bai y Perron (2003), esta prueba asume que se conoce la cantidad de quiebres existentes, pero no deja de ser válido asumir valores de $l$ y realizar ambos tipos de pruebas o una doble maximización.

Sin embargo, las pruebas anteriores no son robustas frente a un modelo con heteroscedasticidad, por lo cual se desarrollaron modelos que superaran esta limitante, uno de ellos es la prueba MZ utilizada por Maasoumi, Zaman y Ahmed (2010). El estadístico MZ tiene más potencia que otras pruebas al ser la suma de dos ratios de verosimilitud que, en su versión simplificada para un único cambio estructural, se define como se muestra en el ecuación [5]:

$$
M Z=(N-k) \log \sigma^{2}-\left[\left(N_{1}-k\right) \log \sigma_{1}^{2}+\left(N_{2}-k\right) \log \sigma_{2}^{2} .\right.
$$

Donde $N$ representa el número de observaciones de la población y $N_{i}$ corresponde al número de observaciones de la muestra $i$; $\sigma^{2}$ es la varianza para el modelo completo y el subíndice indica la submuestra a la cual cada varianza corresponde; finalmente, $k$ es el número de variables explicativas del modelo que es el mismo para ambas submuestras.

La hipótesis nula bajo este estadístico es la estabilidad tanto de los estimadores como de la varianza, y la hipótesis alterna es la inestabilidad tanto en los estimadores como en la varianza de cada submuestra, es decir, $H o: \beta_{1}=\beta_{2}, \sigma_{1}^{2}=\sigma_{2}^{2}$ contra $H a: \beta_{1} \neq \beta_{2}, \sigma_{1}^{2} \neq \sigma_{i}$.

Ante la necesidad de conocer la fecha de cambio en los parámetros, Ahmed, Haider y Zaman (2017), siguiendo lo propuesto por Andrews (1993), realizaron varias iteraciones para diferentes tamaños de submuestras y seleccionar el valor MZ más alto, según se muestra en la ecuación [6].

$$
\operatorname{Sup} M Z=\operatorname{máx} .\left(M Z_{j}\right) ; \quad k<a \leq j \leq b<T-
$$


La ecuación [6] indica que el posible cambio de estructura dentro de la serie de tiempo se da en el valor máximo de MZ tomado de un vector de estadísticos $\mathrm{MZ}$, construidos a partir de diferentes tamaños de submuestras comprendidas entre $a$ y 6 , siendo estos los límites que menciona Andrews (1993) para que las submuestras tengan valores suficientes que no permitan un sesgo por contar con muy pocas observaciones.

Respecto a la prueba de hipótesis sobre el estadístico resultante de la ecuación [6], Ahmed, Haider y Zaman (2017) proponen la realización de bootstraping para obtener los valores críticos en la medida en que se trata de un método no paramétrico y susceptible a los datos utilizados. No obstante, al tratarse de series de tiempo, los datos presentan una estructura particular (tendencias, ciclos o estacionalidad) que puede llegar a omitirse al usar técnicas de bootstraping que asuman independencia en los datos. Para ello, se recurre al bootstraping estacionario explicado en detalle por Lahiri (2003). La idea principal es realizar el bootstraping por bloques de longitud aleatoria. Esto permite que el muestreo realizado no tome cada observación como independiente, sino que conserve, al menos en parte, la estructura de dependencia temporal de la serie.

\section{METODOLOGÍA}

Las variables para llevar a cabo la investigación son los índices bursátiles y el volumen respectivo de los países que conforman el MILA. En particular, se usó el precio de cierre de cada índice en el periodo comprendido entre enero de 2007 y marzo de 2019, lo que dio el total de 2.911 observaciones en promedio por índice. Para el Colcap, específicamente se tomó la serie desde septiembre de 2007 al no contar con información anterior a la fecha. A su vez, se contó con la serie histórica del índice Dow Jones para el mismo periodo, la cual es utilizada como variable exógena en los modelos univariados. La fuente principal de información es Economatica ${ }^{\circledR}$.

Las series de tiempo mencionadas se transformaron en retornos logarítmicos diarios. Posteriormente, se estimaron modelos econométricos univariados capaces de explicar los retornos, incluyendo variables exógenas'. La estimación se puede ver como auxiliar en la medida en que no es necesario garantizar todos los supuestos tradicionales ${ }^{2}$, dado que el propósito es determinar la robustez de las pruebas ante la

1 Durante el proceso de estimación fue necesario ensayar diferentes especificaciones y variables exógenas como: el precio del petróleo, tasas de cambio, entre otras. No obstante, el índice Dow Jones mostró los mejores resultados.

2 No autocorrelación, homocedasticidad y normalidad. 
presencia de autocorrelación y heterocedasticidad. No obstante, se utilizaron los criterios de información de Akaike y Schwarz para definir el modelo más parsimonioso.

Una vez identificado y estimado el modelo univariado se procedió a la detección de los cambios estructurales mediante las pruebas presentadas en la sección anterior. Para ello, se utilizó el software E-views para las pruebas Quandt-Andrews Unknown Breakpoint y las pruebas de cambio secuencial y global de Bai y Perron, (Sequential Lbreaks vs. L. Sequential all Sub-sets, Global vs. none, y L+1 vs. Global). En el caso del estadístico sup MZ y las respectivas pruebas de hipótesis fue necesario crear una rutina en el paquete estadístico RStudio ${ }^{3}$. En particular, para estimar los valores críticos del sup $\mathrm{MZ}$ se siguió el siguiente procedimiento:

1. Se aplica el bootstrap estacionario para la variable dependiente y explicativas en el modelo univariado.

2. Se aplica el procedimiento para hallar el sup MZ.

3. Se replican cinco mil veces los pasos 1 y 2 .

4. Se construye la distribución del sup MZ con las cinco mil observaciones del paso 3.

5. Se calculan los valores críticos para los niveles de significancia de $1 \%, 5 \%$ y $10 \%$.

6. Se repiten los pasos para cada modelo univariado.

Los resultados de las pruebas se contrastaron entre sí para determinar si el cambio se da en el estimador de la media, la varianza o en ambos. Finalmente, se hace un breve análisis de los eventos que tuvieron lugar en las fechas propuestas para los cambios estructurales.

\section{RESULTADOS}

\subsection{Modelos univariados}

Los modelos seleccionados para los retornos de cada uno de los índices tomados cumplieron con las condiciones necesarias para la aplicación de las pruebas de cambio estructural. En la tabla 1 se puede apreciar que todos los modelos tienen como variable exógena el retorno del índice Dow Jones industrial en el mismo día y en un rezago, así como diferentes rezagos de la variable.

3 Las rutinas desarrolladas se pueden solicitar a los autores. 
Ninguno de los modelos cumplió con la normalidad de los errores ni con la condición de heterocedasticidad validada a través de la prueba de BreuschPagan-Godfrey, la prueba de White y la prueba de Harvey, sin embargo, el modelo del retorno del Colcap cumplió con la primera y tercera de las pruebas anteriormente mencionadas.

Tabla 1. Modelos para el retorno de los índices

\begin{tabular}{|c|c|c|c|c|}
\hline Regresor & R_Colcap & R_IPSA & R_IPC & R_SPBL \\
\hline R_DOW & $\begin{array}{c}0,366 \\
{[23,69]^{* * *}}\end{array}$ & $\begin{array}{c}0,473 \\
{[35,69]^{* * *}}\end{array}$ & $\begin{array}{c}0,738 \\
{[56,38]^{* * *}}\end{array}$ & $\begin{array}{c}0,598 \\
{[29,34]^{* * *}}\end{array}$ \\
\hline R_DOW $(-1)$ & $\begin{array}{c}0,181 \\
{[11,68]^{* * *}}\end{array}$ & $\begin{array}{c}0,134 \\
{[8,71]^{* * *}}\end{array}$ & $\begin{array}{c}0,081 \\
{[4,34]^{* * *}}\end{array}$ & $\begin{array}{l}0,194 \\
{[8,44]}\end{array}$ \\
\hline R_Colcap $(-4)$ & $\begin{array}{l}-0,036 \\
{[-2,1]^{* *}}\end{array}$ & & & \\
\hline R_Colcap (-24) & $\begin{array}{c}0,043 \\
{[2,53]^{* *}}\end{array}$ & & & \\
\hline R_IPSA $(-1)$ & & $\begin{array}{c}0,109 \\
{[6,14]^{* * *}}\end{array}$ & & \\
\hline R_IPSA(-3) & & $\begin{array}{c}-0,058 \\
{[-3,86]^{* * *}}\end{array}$ & & \\
\hline R_IPC(-1) & & & $\begin{array}{c}0,076 \\
{[4,15]^{* * *}}\end{array}$ & \\
\hline R_IPC(-2) & & & $\begin{array}{c}-0,033 \\
{[-2,52]^{* *}}\end{array}$ & \\
\hline R_IPC( $(-3)$ & & & $\begin{array}{c}-0,051 \\
{[-4,02]^{* * *}}\end{array}$ & \\
\hline R_SPBL $(-1)$ & & & & $\begin{array}{c}0,149 \\
{[8,19]^{* * *}}\end{array}$ \\
\hline R_SPBL $(-2)$ & & & & $\begin{array}{c}-0,035 \\
{[-2,13]^{* *}}\end{array}$ \\
\hline R_SPBL $(-3)$ & & & & $\begin{array}{c}0,049 \\
{[3,05]^{* * *}}\end{array}$ \\
\hline R_SPBL $(-4)$ & & & & $\begin{array}{c}0,063 \\
{[3,91]^{* * *}}\end{array}$ \\
\hline R_SPBL $(-15)$ & & & & $\begin{array}{c}0,038 \\
{[2,44]^{* *}}\end{array}$ \\
\hline $\mathrm{R}^{2}$ & 0,197 & 0,325 & 0,523 & 0,271 \\
\hline
\end{tabular}

[ ] t-estadístico. ${ }^{* * *}$ Significativo al $1 \%,{ }^{* *}$ al $5 \%,{ }^{*}$ al $10 \%$.

Fuente: elaboración propia a partir del programa E-views 10.

En el caso de los modelos para la variación porcentual del volumen, también se cumplieron con las condiciones necesarias para la aplicación de las pruebas de cambio estructural. En la tabla 2 se puede apreciar que, con excepción de IPSA, los 
modelos tienen como variable exógena el retorno contemporáneo del índice Dow Jones Industrial.

En cuanto a las condiciones de ruido blanco, ninguno de los modelos cumple con el supuesto de normalidad, pero sí cumplen con el supuesto de no autocorrelación. Respecto a la homocedasticidad, solo el modelo para IPSA cumple con las tres pruebas clásicas (Breusch-Pagan-Godfrey y White). Mientras que los otros índices solo cumplen la homocedasticidad en dos de las tres pruebas.

Tabla 2. Modelos para el cambio porcentual del volumen de los índices

\begin{tabular}{|c|c|c|c|c|}
\hline Regresor & D_Colcap & D_IPSA & D_IPC & D_SPBL \\
\hline Constante & $\begin{array}{c}0,214 \\
{[14,85]^{* * *}}\end{array}$ & $\begin{array}{c}0,177 \\
{[12,05]^{* * *}}\end{array}$ & $\begin{array}{c}0,114 \\
{[12,8]^{* * *}}\end{array}$ & $\begin{array}{c}0,680 \\
{[7,88]^{* * *}}\end{array}$ \\
\hline R_DOW & $\begin{array}{c}2,675 \\
{[2,41]^{* *}}\end{array}$ & & $\begin{array}{c}1,642 \\
{[2,33]^{* *}}\end{array}$ & $\begin{array}{c}30,83 \\
{[4,19]^{* * *}}\end{array}$ \\
\hline D_Colcap $(-1)$ & $\begin{array}{c}-0,283 \\
{[-14,85]^{* * *}}\end{array}$ & & & \\
\hline D_Colcap $(-2)$ & $\begin{array}{c}-0,141 \\
{[-7,21]^{* * *}}\end{array}$ & & & \\
\hline D_Colcap $(-3)$ & $\begin{array}{c}-0,067 \\
{[-3,57]^{* * *}}\end{array}$ & & & \\
\hline D_IPSA $(-1)$ & & $\begin{array}{c}-0,222 \\
{[-12,25]^{* * *}}\end{array}$ & & \\
\hline D_IPSA(-2) & & $\begin{array}{c}-0,092 \\
{[-5,07]^{* * *}}\end{array}$ & & \\
\hline D_IPSA $(-4)$ & & $\begin{array}{c}-0,062 \\
{[-3,49]^{* * *}}\end{array}$ & & \\
\hline D_IPSA(-34) & & $\begin{array}{c}0,054 \\
{[3,04]^{* * *}}\end{array}$ & & \\
\hline $\mathrm{D}_{-} \mathrm{IPC}(-1)$ & & & $\begin{array}{c}-0,322 \\
{[-17,61]^{* * *}}\end{array}$ & \\
\hline$D_{-} \operatorname{IPC}(-2)$ & & & $\begin{array}{c}-0,166 \\
{[-8,66]^{* * *}}\end{array}$ & \\
\hline$D_{-} \operatorname{IPC}(-3)$ & & & $\begin{array}{c}-0,105 \\
{[-5,51]^{* * *}}\end{array}$ & \\
\hline D_IPC(-4) & & & $\begin{array}{c}-0,036 \\
{[-1,98]^{* *}}\end{array}$ & \\
\hline D_SPBL(-1) & & & & $\begin{array}{c}-0,046 \\
{[-2,54]^{* * *}}\end{array}$ \\
\hline $\mathrm{R}^{2}$ & 0,08 & 0,05 & 0,1 & 0,008 \\
\hline
\end{tabular}

[] t-estadístico. ${ }^{* * *}$ Significativo al $1 \%, * *$ al $5 \%, *$ al $10 \%$.

Fuente: elaboración propia a partir del programa E-views 10. 


\subsection{Pruebas de cambio estructural}

A partir de los modelos construidos se procede a realizar las pruebas de cambio estructural contrastadas a un nivel de significancia del $5 \%$. Se hizo un recorte de datos del $15 \%$ en ambos extremos de las series de tiempo con el fin de contar con suficientes datos en cada submuestra para su posterior comparación, a excepción de la serie de tiempo para el Colcap que tuvo un recorte del $10 \%$ a cada extremo, con el fin de llegar al máximo valor tanto en la prueba F iterativa como en la sup MZ.

En la tabla 3 aparecen los resultados de las diferentes pruebas de cambio estructural aplicadas a cada uno de los índices. Las fechas están organizadas en mes, día y año. Asimismo, aparece el contraste de la prueba $\mathrm{F}$ para la fecha detectada por el estadístico sup MZ, el cual indica que, según la prueba de Chow para las fechas detectadas por este último, existe un cambio de estructura en los estimadores.

Tabla 3. Fechas de cambio estructural (CE) para el retorno de los índices

\begin{tabular}{lcccc}
\hline \multicolumn{1}{c}{ Prueba de cambio estructural } & Colcap & IPSA & IPC & SEP/BVL \\
\hline Quandt-Andrews unknown breakpoint & $11 / 20 / 2008$ & $11 / 05 / 2008$ & $4 / 28 / 2010$ & $11 / 24 / 2008$ \\
\hline Sequential L breaks vs. L & $11 / 20 / 2008$ & $11 / 05 / 2008$ & $4 / 28 / 2010$ & $11 / 24 / 2008$ \\
\hline Sequential all sub-sets & $11 / 20 / 2008$ & $11 / 05 / 2008$ & $4 / 28 / 2010$ & $11 / 24 / 2008$ \\
\hline Global vs none & $11 / 19 / 2008$ & $11 / 05 / 2008$ & $4 / 28 / 2010$ & $11 / 24 / 2008$ \\
\hline L + 1 vs global & $11 / 20 / 2008$ & $11 / 05 / 2008$ & $4 / 28 / 2010$ & $11 / 24 / 2008$ \\
\hline MZ & $12 / 16 / 2008$ & $11 / 21 / 2008$ & $02 / 12 / 2009$ & $10 / 27 / 2011$ \\
\hline Contraste: prueba F sobre fecha sup MZ & Sí hay CE & Sí hay CE & Sí hay CE & Sí hay CE \\
\hline
\end{tabular}

Fuente: elaboración propia a partir de los programas E-views 10 y RStudio.

Se aprecia que para cada índice las pruebas de cambio estructural clásicas arrojan la misma fecha, caso contrario al resultado obtenido por el sup MZ. Así, se determina que para el Colcap la diferencia es de veintiséis días, para el IPSA de dieciséis, para el IPC de ciento cuarenta y cinco y para el SEP/BVL de mil sesenta y siete días.

Al realizar el contraste con la prueba de Chow, se evidencia la existencia de un cambio en los estimadores para la fecha detectada por el sup MZ, lo que sugiere que existe un cambio estructural diferente al detectado por las pruebas clásicas, las cuales exigen el cumplimiento de la condición de ruido blanco. Debido a que los modelos no cumplen con dos de las condiciones se establece que los resultados más confiables son aquellos obtenidos por la prueba robusta en presencia de heterocedasticidad y de autocorrelación, es decir, el sup MZ. 
Al realizar la prueba $\mathrm{F}$ de forma individual con el fin de determinar en cuáles estimadores ocurrió el cambio específicamente, se corrobora que para todos los modelos existió modificación de algunos de sus estimadores (anexo 1). Además, se corroboró el cambio para las varianzas de las dos submuestras creadas a partir de la división en la fecha del sup MZ con la prueba de igualdad de varianzas. Los resultados muestran que la varianza cambió para todos los modelos, siendo la varianza antes del cambio estructural mucho mayor (anexo 1).

Por su parte, la significancia estadística del sup MZ se calculó mediante el bootstraping estacionario con cinco mil muestreos para cada una de los modelos multivariados. Según los resultados de la tabla 4, se observa que para Colcap, IPSA e IPC el sup MZ es significativo para un nivel del $5 \%$, mientras que para el S\&P/ BVL es del $1 \%$. Es decir, para todos los índices analizados es posible rechazar la hipótesis nula de que no existe cambio estructural en media y varianza.

Tabla 4. Valores críticos del estadístico sup MZ-. Retornos de los índices accionarios

\begin{tabular}{lcccc}
\hline & Colcap & IPSA & IPC & S\&P/BVL \\
\hline Sup MZ & 359,23 & 206,98 & 260,82 & 716,36 \\
\hline Valor crítico al 1\% & 359,45 & 303,28 & 346,44 & 404,67 \\
\hline Valor crítico al 5\% & 228,17 & 203,19 & 224,55 & 253,17 \\
\hline Valor crítico al 10\% & 176,65 & 156,76 & 172,03 & 192,97 \\
\hline
\end{tabular}

Fuente: elaboración propia a partir del programa RStudio.

La tabla 5 presenta los resultados de cambio estructural para los modelos del cambio porcentual del volumen con el mismo esquema usado para los retornos.

Tabla 5. Fechas de cambio estructural (CE) para el cambio porcentual de los índices

\begin{tabular}{lcccc}
\hline \multicolumn{1}{c}{ Prueba de cambio estructural } & Colcap & IPSA & IPC & SEP/BVL \\
\hline Quandt-Andrews unknown breakpoint & $03 / 18 / 2015$ & No CE & $9 / 04 / 2012$ & No CE \\
\hline Sequential L breaks vs L & $03 / 18 / 2015$ & No CE & $9 / 04 / 2012$ & No CE \\
\hline Sequential all sub-sets & $03 / 18 / 2015$ & No CE & $9 / 04 / 2012$ & No CE \\
\hline Global vs none & $03 / 18 / 2015$ & No CE & $9 / 04 / 2012$ & No CE \\
\hline L +1 vs global & $03 / 18 / 2015$ & No CE & $9 / 04 / 2012$ & No CE \\
\hline MZ & $03 / 17 / 2015$ & $5 / 28 / 2012$ & $8 / 27 / 2012$ & $08 / 29 / 2013$ \\
\hline Contraste: prueba F sobre fecha MZ & Sí hay CE & No CE & Sí hay CE & No CE \\
\hline
\end{tabular}

Fuente: elaboración propia a partir de los programas E-views 10 y RStudio. 
En primer lugar, para los índices IPSA y S\&P/BVL se aprecia cómo los resultados de las pruebas clásicas de cambio estructural no permiten rechazar la hipótesis nula de la no existencia del quiebre. Además, al contrastar la fecha encontrada por el sup MZ con la prueba F tampoco se detecta un cambio estructural. De este modo, se procedió a verificar si la fecha detectada es por un cambio en la varianza o para uno de los estimadores $\beta$ de forma individual (anexo 2). Mediante la prueba de Chow se evidencia que los estimadores no varían entre las dos submuestras, ni global ni individualmente. Por otro lado, al realizar la prueba de igualdad de varianzas se rechaza la hipótesis nula de que estas son iguales. Así, la fecha del sup MZ indica que la variación porcentual del volumen en los índices IPSA y S\&P/ BVL se vio afectada solamente por la volatilidad (anexo 2).

En segundo lugar, para los índices IPC y Colcap se evidencia un comportamiento similar a los modelos de los retornos, es decir, las pruebas clásicas arrojan las mismas fechas de cambio estructural, mientras que el estadístico del sup MZ identifica otra. Se determina que para el índice Colcap la diferencia es de un día, mientras que para el IPC es de ocho días.

$\mathrm{Al}$ igual que en los retornos, se realiza la prueba $\mathrm{F}$ para determinar de forma individual el cambio en los estimadores y la varianza (anexo 2). En este caso, se aprecia que para el Colcap e IPC los modelos muestran un cambio en la constante y sus componentes autorregresivos. En el caso de la varianza, se corroboró que para ambos modelos existió una modificación en la varianza, pues esta fue mayor después del cambio estructural (anexo 2).

La prueba de hipótesis sup MZ se calculó con el mismo método explicado para los modelos de los retornos. La tabla 6 confirma la existencia de un cambio estructural para el Colcap e IPC, para niveles de significancia del $10 \%$ y $5 \%$ respectivamente. Por el contrario, para IPSA y SEP/BVL no se rechaza la hipótesis nula para ningún nivel de confianza.

Tabla 6. Valores críticos del estadístico sup MZ. Cambio porcentual de los índices

\begin{tabular}{ccccc}
\hline & Colcap & IPSA & IPC & SEP/BVL \\
\hline Sup MZ & 243,35 & 205,59 & 277,54 & 283,87 \\
\hline Valor crítico al 1\% & 362,59 & 577,62 & 378,37 & $1.577,47$ \\
\hline Valor crítico al 5\% & 245,72 & 401,36 & 263,06 & $1.206,64$ \\
\hline Valor crítico al 10\% & 199,77 & 331,12 & 209,54 & $1.007,82$ \\
\hline
\end{tabular}

Fuente: elaboración propia a partir del programa RStudio. 


\subsection{Discusión}

\subsubsection{Colombia}

La fecha detectada por el sup MZ (16 de diciembre de 2008) se encuentra dentro del periodo de mayor volatilidad de la tasa de cambio que, según El Tiempo (2009), fue ocasionada en mayor medida por la crisis del 2008 que generó una alta volatilidad en los mercados. Así, la disminución de la varianza en la segunda submuestra puede ser explicada por las medidas tomadas por los bancos centrales para dinamizar la economía global, como lo fue la disminución de las tasas de interés de la Federal Reserve Board (FED) que, de acuerdo con Pozzi (2008), buscaba aliviar la caída del empleo y la disminución de la producción industrial en Estados Unidos. El anuncio fue realizado el 16 de diciembre de 2008 en una nota de prensa, fecha en la cual el estadístico MZ marcó el cambio de estructura.

Respecto al volumen, la fecha calculada por el sup MZ (17 de marzo de 2015) coincide con noticias como la caída del precio del petróleo WTI un día antes de la fecha estimada hasta su nivel más bajo en nueve años, para luego estabilizarse alrededor de ese precio en los años siguientes. Según El Tiempo (2015), la caída es atribuida al exceso de oferta en el mercado dado el aumento en la producción de Estados Unidos. Esto ocasionó una fuerte caída en los precios de las acciones de Pacific Rubiales, Canacol Energy y Ecopetrol, lo que explicaría la disminución en la capitalización bursátil y la manera en que el cambio en los precios del petróleo y el dólar afectaron la estructura del modelo para la variación porcentual del volumen del índice Colcap.

\subsubsection{Chile}

La fecha indicada por el sup MZ (21 de noviembre de 2008) para el retorno del IPSA se cruza con eventos de índole internacional como la cumbre del G-20, realizada cinco días antes. El País (2008a) argumenta que en esta cumbre se plantearon las medidas necesarias en cuanto a la regulación financiera para impedir una futura crisis como la del 2008. Tres días después de los anuncios, El País (2008b) explica que los mercados financieros de todo el mundo sufrieron una caída generalizada ante las malas perspectivas de crecimiento de los Estados Unidos hechas por la FED. Las acciones de las entidades financieras fueron las más perjudicadas.

En cuanto a la fecha para el cambio porcentual del volumen del IPSA, es pertinente mencionar que no se detectó un cambio en la media, sino solamente en la varianza. Este incremento de la volatilidad coincide con una ampliación del mercado 
de renta que permitiría a los inversores locales comprar acciones norteamericanas en la bolsa de Santiago en acuerdo con el Deutsche Bank.

\subsubsection{México}

La fecha identificada por el sup MZ para el retorno del IPC (12 de febrero de 2009) no coincide con ningún evento de importancia nacional o internacional que respalde el cambio de estructura. Sin embargo, con un análisis en fechas cercanas se encuentra una combinación de sucesos de impacto para la economía mexicana. Primero, según Reuters (2009), el aumento del IVA y del impuesto sobre la renta ahuyentó a los inversionistas. A su vez, según Blanke (2009), México fue el país latinoamericano más afectado por la crisis debido a su dependencia de los Estados Unidos. Cuando a inicios de diciembre del 2009 se publicaron los datos macroeconómicos de la mejora en la economía de los Estados Unidos, la bolsa de valores de México experimentó una notable mejora impulsada por Wall Street.

En cuanto al volumen (27 de agosto de 2012) se encontró que el único evento de importancia fue el que reportó El País (2012) relacionado con el hallazgo del primer gran yacimiento de petróleo en aguas profundas por parte de Pemex. Este podría corresponder a un tercio de la actual producción anual de crudo en el país, siendo las exportaciones de petróleo la principal fuente de ingresos del Estado mexicano, este hallazgo supuso una mejora para las finanzas del país.

\subsubsection{Perú}

Después de una búsqueda exhaustiva de artículos de prensa digital en fechas cercanas a la detectada por el sup MZ para el retorno del índice SEP/BVL (27 de octubre de 2011), no se encontró ningún evento de impacto nacional capaz de explicar el cambio de estructura. Sin embargo, de acuerdo con Portafolio (2011), para esa fecha se da la reducción de la deuda a Grecia en un 50 \% por parte de la Unión Europea. Este suceso marca un hito importante de cara a la crisis que la eurozona estaba pasando y que, según Parodi (2012), comenzó en el 2010 y para la fecha tiene una combinación de elementos que podrían agravar su desempeño y dificultar su recuperación. La relación de la economía europea con Perú es muy fuerte si se tiene en cuenta que Europa fue el destino del $33 \%$ de las exportaciones peruanas en el $2011^{4}$, siendo Suiza el segundo país que más importa productos peruanos después de China.

(https://oec.world/es/visualize/tree_map/hs92/export/per/show/all/2011/) 
Para la variación porcentual en el volumen se encuentra que la fecha (29 de agosto de 2013) está muy próxima a un evento de gran peso para la economía latinoamericana, esto es, la finalización de las negociaciones de la Alianza del Pacífico. Según El Comercio (2013), en estas negociaciones los cuatro socios (Chile, Colombia, Perú y México) acordaron la desgravación del 92 \% de las partidas arancelarias de forma inmediata una vez comenzara el acuerdo, y la desgravación del $8 \%$ restante de forma gradual. Además, Portafolio (2013) comenta que, de acuerdo con estimaciones de Proexport Colombia, las expectativas de crecimiento en las exportaciones provenientes de Perú serían del 22 \% una vez empiece a funcionar el acuerdo que facilitaría el tránsito de mercancías entre los países socios.

\section{CONCLUSIONES}

La detección de un cambio estructural en una fecha concreta resulta de suma importancia para determinar ante qué situaciones un modelo econométrico de regresión lineal debe ser nuevamente estimado con la inclusión del cambio, permitiendo de este modo, se obtienen resultados más confiables y modelos más sólidos que respondan a las características específicas de las variables económicas.

Los resultados de la búsqueda en artículos de prensa para las fechas encontradas por el sup MZ muestran que para los modelos del retorno de cada uno de los índices bursátiles, los eventos que ocasionan un quiebre en la estructura son aquellos originados en las economías desarrolladas. Ante estos los países pertenecientes al MILA se preparan teniendo como indicadores del desempeño global los mercados de los países desarrollados.

Para el caso de Colombia, Chile y México, el quiebre se relaciona más con el desempeño de la economía de Estados Unidos. En Perú, el panorama es similar, con la diferencia de que su principal socio comercial comienza un periodo de crisis en lugar de estar en plena recuperación. Así, los retornos de los índices que conforman el MILA son más sensibles a cambios en la estructura económica, política y financiera de los países desarrollados, que afectan los mercados internacionales, que a eventos de alcance puramente local. Esto puede ser consecuencia de que todos los modelos están atados al índice Dow Jones industrial, utilizado generalmente como indicador de referencia internacional.

Las fechas de cambio estructural para los modelos de la variación porcentual en el volumen de cada uno de los índices bursátiles, indican un quiebre en estos modelos ante la ocurrencia de un evento que tiene relación directa con la economía local de cada país. En Colombia el cambio se da ante la caída de los 
precios del petróleo que se estancan a partir de esa fecha, lo que hace que la capitalización bursátil del país disminuya ante la caída en los precios de las acciones de emisores del sector minero e hidrocarburos, importantes industrias para la economía colombiana. En Chile el cambio ocurre en la fecha en la cual el mercado de renta variable se amplía para permitir a los inversores locales acceder a títulos listados en los Estados Unidos. Para México el cambio se origina frente a las expectativas de los inversores por las mejores calificaciones crediticias de Fitch para las entidades financieras, y el descubrimiento de un pozo de petróleo en el golfo de México, uno de los productos más importantes en la economía mexicana. En Perú el cambio se da con la firma del acuerdo en la Alianza del Pacifico que desgrava el $92 \%$ de las partidas arancelarias de forma inmediata lo que permite que los productos peruanos lleguen más fácil a los mercados vecinos.

Asimismo, se observó que los modelos que no cumplieron con la condición de homocedasticidad mostraron las diferencias más amplias entre las fechas detectadas por las pruebas clásicas y las fechas detectadas por el estadístico MZ. Los modelos de los retornos tuvieron mayor discrepancia. En contraposición, se puede decir que ninguno de estos modelos cumplió con la prueba de homocedasticidad de White a diferencia de los modelos para la variación porcentual del volumen.

Se esperaba la ocurrencia de un cambio estructural en la fecha en la cual fue puesto en marcha el esquema del MILA. Sin embargo, se aprecia que los modelos construidos responden más a las modificaciones en la política económica y a las alteraciones fuertes en los precios de las materias primas. Para el caso peruano es pertinente anotar que respalda la teoría de la integración como una causa de cambio estructural, al existir un quiebre en uno de sus modelos en la fecha en la cual se firma el acuerdo comercial de desgravación de las partidas arancelarias en la Alianza del Pacífico. Por tanto, se recomienda ampliar la investigación para identificar posibles cambios de estructura de diferentes modelos frente a integraciones económicas importantes, como la creación de la eurozona o Mercosur o por la desvinculación de una integración como el Brexit.

\section{BIBLIOGRAFÍA}

Ahmed, Mumtaz; Haider, Gulfan y Zaman, Asad (2017). Detecting structural change with heteroskedasticity. En: Communications in Statistics - Theory and Methods, vol. 46, n. ${ }^{\circ}$ 21, p. 10446-10455. DOI: 10.1080/03610926.2016.1235200

Anderson, Gordon y Mizon, Grayham (1989). What Can Statistics Contribute to the Analysis of Economic Structural Change? En: Hackl, Peter (Ed.). Statistical Analysis and Forecasting of Economic Structural Change. Berlín: Springer, p. 3-21. 
Andrews, Donald (1993). Tests for Parameter Instability and Structural Change with Unknown Change Point. En: Econometrica, vol. 61, n. ${ }^{\circ} 4$, p. 821-856. DOI: 10.2307/2951764

Bai, Jushan y Perron, Pierre (1998). Estimating and Testing Linear Models with Multiple Structural Changes. En: Econometrica, vol. 68, n. ${ }^{\circ}$ 1, p. 47-78. DOI: 10.2307/2998540

Bai, Jushan y Perron, Pierre (2003). Computation and analysis of multiple structural change models. En: Journal of Applied Econometrics, vol. 18, n. ${ }^{\circ}$ 1, p. 1-22. DOI: 10.1002/jae.659

Blanke, Svenja (2009). México: una gran crisis sin (grandes) respuestas. https://nuso.org/articulo/ mexico-una-gran-crisis-sin-grandes-respuestas/

Chow, Gregory (1960). Tests of Equality Between Sets of Coefficients in Two Linear Regressions En: Econometrica, vol. 28, n. 3, p. 591-605. DOI: 10.2307/1910133

El Comercio (2013). Países de la Alianza del Pacífico concluyen negociaciones económicas, Perú. https://bit.ly/2WUVqqo

El País (2008a). La cumbre de Washington se cierra con un compromiso para la reforma de los mercados, España. https://bit.ly/3bx5g6U

El País (2008b). La FED empeora las perspectivas sobre la economía de EE UU, España. https:// elpais.com/economia/2008/11/19/actualidad/1227083580_850215.html

El País (2012). México encuentra su primer gran yacimiento de petróleo en agua profunda, España. https://elpais.com/internacional/2012/08/30/mexico/1346280499_102315.html

El Tiempo (2009). Volatilidad del dólar en 2008 fue la más alta de la historia, Colombia. https:// www.eltiempo.com/archivo/documento/MAM-3258651

El Tiempo (2015). El petróleo alcanzó este lunes el nivel más bajo en los últimos 9 años, Colombia. https://www.eltiempo.com/archivo/documento/CMS-15410459

Hansen, Bruce (2001). The New Econometrics of Structural Change: Dating Breaks in U.S. Labor Productivity. En: Journal of Economic Perspectives, vol. 15, n. ${ }^{\circ} 4$, p. 117-128. DOI: 10.1257/ jep. 15.4.117

Hillebrand, Eric (2005). Neglecting parameter changes in GARCH models. En: Journal of Econometrics, vol. 129, n. ${ }^{\circ} 1-2$, p. 121 138. DOI: 10.1016/j.jeconom.2004.09.005.

Lahiri, Soumendra (2003). Resampling Methods for Dependent Data. Nueva York: Springer, 374p.

MacKinnon, James (1988). Heteroskedasticity-Robust Tests for Structural Change. En: Empirical Economics. vol. 14, p. 1-14. DOI: 10.1007/BF01980588

Maasoumi, Esfandiar; Zaman, Asad y Ahmed, Mumtaz (2010). Tests for structural change, aggregation, and homogeneity. En: Economic Modelling, vol. 27, n. ${ }^{\circ}$ 6, p. 1382-1391. DOI: 10.1016/j.econmod.2010.07.009

Narayan, Paresh; Narayan Seema y Mishra, Sagarika (2013). Has the structural break slowed down growth rates of stock markets? En: Economic Modelling, vol. 30, p. 595-601. DOI: 10.1016/j.econmod.2012.10.001.

Parodi, Carlos (2012). Crisis europea. Perú. https://bit.ly/2WuY6vZ 
Pérez, Julián (1995). Tratamiento econométrico del cambio estructural: el método de estimación paramétrica ponderada. Tesis para optar al título de doctor en Economía. Doctorado en Economía, Universidad Autonoma de Madrid, España, 316 p.

Portafolio (2011). Unión Europea le rebaja la mitad de la deuda a Grecia, Colombia. https://bit. ly/2T2TLxP

Portafolio (2013). Chile, el más beneficiado en la ronda de la Alianza Pacífico, Colombia. https:// bit.ly/2T2OmGO

Pozzi, Sandro (2008). EE. UU. baja los tipos de interés casi al 0 \%, España. https://bit.ly/3bx8J5q

Quandt, Richard (1958). The Estimation of the Parameters of a Linear Regression System Obeying Two Separate Regimes. En: Journal of the American Statistical Association, vol. 53, n. 284 , p. $873-880$. DOI: $10.230 \overline{7 / 2281957 .}$

Reuters (2009). Senado mexicano aprueba alza IVA al 16 pct a partir del 2010, México. https:// lta.reuters.com/articulo/mexico-reforma-iva-idMXN3049846820091030

Shahani, Rakesh y Subhan, Muhammad (2018). Did Brexit Lead to a Structural Break in Stock Returns of Select EU Countries? - A Time Series Econometric Investigation. En: IUP Journal of Financial Risk Management, vol. 15, n. ${ }^{\circ}$ 2, p. 22-36.

Turtle, Harry y Zhang, Chengping (2014). Structural breaks and portfolio performance in global equity markets. En: Quantitative Finance, vol. 15, n. ${ }^{\circ}$ 6, p. 909-922. 
Nicolás Morales León y José Rodrigo Vélez Molano

\section{ANEXO 1}

Tabla A.1.1. Estimadores para el retorno del Colcap

\begin{tabular}{ccccc}
\hline & Muestra completa & Primera submuestra & Segunda submuestra & Significancia individual \\
\hline R_DOW & 0,36643642 & 0,36950181 & 0,37739937 & Sí \\
\hline R_DOW-1 & 0,18138925 & 0,31129251 & 0,10459545 & No \\
\hline R_Colcap-4 & $-0,03616380$ & $-0,05201619$ & $-0,02345892$ & No \\
\hline R_Colcap-24 & 0,04342466 & 0,05672733 & 0,02466220 & No \\
\hline$\sigma^{2}$ & 0,00009208 & 0,00027482 & 0,00006858 & Sí \\
\hline
\end{tabular}

Fuente: elaboración propia a partir del programa RStudio.

Tabla A.1.2. Estimadores para el retorno del IPSA

\begin{tabular}{ccccc}
\hline & Muestra completa & Primera submuestra & Segunda submuestra & Significancia individual \\
\hline R_DOW & 0,47302172 & 0,58153926 & 0,41978585 & Sí \\
\hline R_IPSA-1 & 0,10872985 & 0,05451093 & 0,12563224 & No \\
\hline R_IPSA-3 & $-0,05837490$ & $-0,07816609$ & $-0,05254425$ & No \\
\hline R_DOW-1 & 0,13478397 & 0,23957078 & 0,09481618 & Sí \\
\hline$\sigma^{2}$ & 0,00007037 & 0,00014203 & 0,00005613 & Sí \\
\hline
\end{tabular}

Fuente: elaboración propia a partir del programa RStudio.

Tabla A.1.3. Estimadores para el retorno del IPC

\begin{tabular}{ccccc}
\hline & Muestra completa & Primera submuestra & Segunda submuestra & Significancia individual \\
\hline R_DOW & 0,73856120 & 0,85095727 & 0,61759476 & Sí \\
\hline R_IPC-1 & 0,08135452 & 0,18133666 & 0,01309754 & Sí \\
\hline R_IPC-2 & 0,07578307 & 0,04253410 & 0,07210987 & No \\
\hline R_IPC-3 & $-0,03267213$ & $-0,01496100$ & $-0,02731042$ & No \\
\hline R_DOW-1 & $-0,05132991$ & $-0,05784569$ & $-0,05828157$ & Sí \\
\hline$\sigma^{2}$ & 0,00006930 & 0,00011866 & 0,00005147 & Sí \\
\hline
\end{tabular}

Fuente: elaboración propia a partir del programa RStudio.

Tabla A.1.4. Estimadores para el retorno del S\&P/BVL

\begin{tabular}{ccccc}
\hline & Muestra completa & Primera submuestra & Segunda submuestra & Significancia individual \\
\hline R_DOW & 0,59775972 & 0,68435934 & 0,42091719 & Sí \\
\hline R_DOW-1 & 0,19387646 & 0,26510947 & 0,06592143 & Sí \\
\hline R_SPBL-1 & 0,14851821 & 0,14052907 & 0,15450040 & No \\
\hline
\end{tabular}


Cambios estructurales en índices bursátiles del mercado MILA entre los años 2008 y 2018

\begin{tabular}{ccccc}
\hline & Muestra completa & Primera submuestra & Segunda submuestra & Significancia individual \\
\hline R_SPBL-2 & $-0,03533303$ & $-0,03220970$ & $-0,01251581$ & No \\
\hline R_SPBL-3 & 0,04972273 & 0,05483848 & 0,01683524 & No \\
\hline R_SPBL-4 & 0,06279625 & 0,07766620 & 0,01056067 & No \\
\hline R_SPBL-15 & 0,03849695 & 0,03742700 & 0,01994276 & Sí \\
\hline$\sigma^{2}$ & 0,00016408 & 0,00029962 & 0,00007207 & Sí \\
\hline
\end{tabular}

Fuente: elaboración propia a partir del programa RStudio.

\section{ANEXO 2}

Tabla A.2.1. Estimadores para la variación porcentual del volumen del índice Colcap

\begin{tabular}{ccccc}
\hline & Muestra completa & Primera submuestra & Segunda submuestra & Significancia individual \\
\hline C & 0,21488198 & 0,17251706 & 0,30731247 & Sí \\
\hline R_DOW & 2,67527149 & 2,01444891 & 5,37450715 & No \\
\hline D_Colcap-1 & $-0,28293503$ & $-0,28578988$ & $-0,29695294$ & No \\
\hline D_Colcap-2 & $-0,14137214$ & $-0,17536161$ & $-0,12965007$ & Sí \\
\hline D_Colcap-3 & $-0,06758665$ & $-0,08823523$ & $-0,06456702$ & No \\
\hline$\sigma^{2}$ & 0,4830717 & 0,3327567 & 0,7589435 & Sí \\
\hline
\end{tabular}

Fuente: elaboración propia a partir del programa RStudio.

Tabla A.2.2. Estimadores para la variación porcentual del volumen del índice IPSA

\begin{tabular}{ccccc}
\hline & Muestra completa & Primera submuestra & Segunda submuestra & Significancia individual \\
\hline C & 0,17698778 & 0,16303037 & 0,19186941 & No \\
\hline D_IPSA-1 & $-0,22220219$ & $-0,27563033$ & $-0,20350743$ & No \\
\hline D_IPSA-2 & $-0,09211991$ & $-0,10511086$ & $-0,08992864$ & No \\
\hline D_IPSA-4 & $-0,06208426$ & $-0,07497671$ & $-0,05906704$ & No \\
\hline D_IPSA-34 & 0,05419118 & 0,03841322 & 0,06152573 & No \\
\hline$\sigma^{2}$ & 0,5719848 & 0,346156 & 0,7481492 & Sí \\
\hline
\end{tabular}

Fuente: elaboración propia a partir del programa RStudio. 
Nicolás Morales León y José Rodrigo Vélez Molano

Tabla A.2.3. Estimadores para la variación porcentual del volumen del índice IPC.

\begin{tabular}{ccccc}
\hline & Muestra completa & Primera submuestra & Segunda submuestra & Significancia individual \\
\hline C & 0,11443477 & 0,11201830 & 0,12718891 & Sí \\
\hline R_DOW & 1,64209005 & 2,37532856 & $-0,68619377$ & No \\
\hline D_IPC-1 & $-0,32258928$ & $-0,39689845$ & $-0,30468230$ & No \\
\hline D_IPC-2 & $-0,16582556$ & $-0,28256644$ & $-0,12892144$ & Sí \\
\hline D_IPC-3 & $-0,10553115$ & $-0,24702119$ & $-0,06015160$ & Sí \\
\hline D_IPC-4 & $-0,03633523$ & $-0,08049201$ & $-0,03152807$ & No \\
\hline$\sigma^{2}$ & 0,2028075 & 0,1164174 & 0,2741844 & Sí
\end{tabular}

Fuente: elaboración propia a partir del programa RStudio.

Tabla A.2.4. Estimadores para la variación porcentual del volumen del índice S\&P/BVL

\begin{tabular}{ccccc}
\hline & Muestra completa & Primera submuestra & Segunda submuestra & Significancia individual \\
\hline C & 0,68057126 & 0,71625597 & 0,64725775 & No \\
\hline R_DOW & 30,8348030 & 34,6597443 & 18,8251162 & No \\
\hline SEP/BVL-1 & $-0,04643240$ & $-0,03921308$ & $-0,06344122$ & No \\
\hline$\sigma^{2}$ & 21,6884000 & 29,6422600 & 12,1376800 & Sí \\
\hline
\end{tabular}

Fuente: elaboración propia a partir del programa RStudio. 